\title{
Knowledge, Attitude and Practice of the Community towards Malaria Prevention and Control Options in Anti-Malaria Association Intervention Zones of Amahara National Regional State, Ethiopia
}

\section{Zewdie Aderaw $^{1 *}$ and Molla Gedefaw ${ }^{2}$}

${ }^{1}$ Public Health Department, College of Health Sciences, Debre Markos University, Ethiopia

${ }^{2}$ Public health department, College of medicine, Bahir Dar University, Ethiopia

\section{Abstract}

Background: Malaria is one of the most important public health challenges in Ethiopia. Although the disease is endemic in many parts of the country, the knowledge, attitude and practice of the community about the disease prevention and control options is far from perfect, and misconceptions and malpractices are common.

Objective: To assess the level of knowledge, attitude and practices of the community towards malaria prevention and control options.

Methodology: A community based cross sectional study design was done in Antimaria Association intervention zones. A single population proportion sample size formula and design effect of two was used to determine sample size. A total of 864 participants were included in the study and proportional allocation was done among urban and rural residents. The data was collected by trained data collectors and supervisors using questionnaires and interviewing guidelines. The collected data was cleaned, coded and entered into SPSS version 16.0 for windows soft ware for analysis.

Results: This study revealed that $37.6 \%$ of the study participants mentioned fever as symptom of malaria. The acceptance rate of IRS as malaria control and prevention method is $5.37 \%$. From the general population, $26.4 \%$ of the participants used ITN as malaria prevention and control method. Among febrile children in the last one year, $28.4 \%$ were taken to modern health care institutions for treatment. From the total study participants, $66.6 \%, 50.8 \%$, $64.8 \%$ have a good knowledge on clinical manifestations, signs and symptoms, and prevention methods of malaria, respectively. Sixty nine percent of the respondents have positive attitude towards modern health care utilization for malaria treatment and $47 \%$ of them have good practice towards malaria prevention and control activities.

Conclusion and recommendation: knowledge, attitude and practice of the community towards malaria prevention and control options are still at low level. Therefore, the existing effort must be strengthened and continued to improve the community knowledge, attitude and practice towards malaria prevention and control options.

Keywords: Malaria; Knowledge; Attitude; Practice; Insecticide treated net utilization

\section{Introduction}

Malaria affects the health and wealth of nations and individuals alike. In Africa today, malaria is understood to be both a disease of poverty and a cause of poverty. Annual economic growth in countries with high malaria transmission has historically been lower than in countries without malaria. Economists believe that malaria is responsible for a 'growth penalty' of up to $1.3 \%$ per year in some African countries [1]. In Ethiopia, malaria is at the forefront among the health problems of the country. The actual number of malaria cases that occur annually throughout the country are estimated to be about 4-5 million. Due to climatic and geographic factors, the disease occurs in different parts of the country in epidemic form. About $75 \%$ of the total area of the country is estimated to be malarious wherein $68 \%$ of the total populations live, 40 million people, being at risk of infection [2].

Studies indicated that despite these bitter facts, communities are not well aware of the multi-dimensional challenges of malaria in our country. The knowledge of the community is far from perfect, and misconceptions are rampant [2]. There have been a considerable number of reports about knowledge, attitude, and practice relating to malaria and its control from different parts of Africa. These reports concluded that misconceptions concerning malaria still exist and that practices for the control of malaria have been unsatisfactory [3].
Ethiopian national malaria indicator survey of 2007 indicated that about $71 \%$ of the rural community and $80.5 \%$ of the urban community have heard about malaria. This survey also reported that about $87 \%$ of Amhahara national regional state respondents had heard about malaria. However, only $30.1 \%$ of the rural and $59.7 \%$ of urban community knew that mosquito bite can transmit malaria. This survey indicated that $52.7 \%$ of pregnant women, $51.8 \%$ of children have slept under ITN in the previous day of the interview. Thus, understanding of the current knowledge of the community beliefs and practices with respect to the disease is required to obtain and maintain the community involvement in surveillance and control activities [3].

Many reports indicated, currently the burden of malaria is

*Corresponding author: Zewdie Aderaw, Public Health Department, College of Health Sciences, Debre Markos University, Ethiopia, Tel: 251911550926 E-mail: Zewdie1984@gmail.com

Received July 03, 2013; Accepted September 04, 2013; Published September 07, 2013

Citation: Aderaw Z, Gedefaw M (2013) Knowledge, Attitude and Practice of the Community towards Malaria Prevention and Control Options in Anti-Malaria Association Intervention Zones of Amahara National Regional State, Ethiopia. J Trop Dis 1: 118. doi:10.4172/2329-891X.1000118

Copyright: ( 2013 Aderaw Z, et al. This is an open-access article distributed unde the terms of the Creative Commons Attribution License, which permits unrestricted use, distribution, and reproduction in any medium, provided the original author and source are credited. 
decreasing in the country with the government different prevention and control strategies. For the sustainability of the programs, capacity building must be done on the community knowledge, attitude and practice towards malaria prevention and control options. However, the attention of most researchers on the topic is reducing from time to time. Therefore, this study was designed to assess the current level of knowledge, attitude and practice of the community towards malaria prevention and control options. The output of this study is very important for the sustainability of the current malaria prevention and control options through assessing the current community knowledge, attitude and practice of malaria prevent and control options.

\section{Methods}

\section{Research design}

A community based descriptive cross sectional study design was used to assess knowledge, attitude and practice of the community towards malaria prevention and control options.

\section{Study area and study period}

The study was conducted in five intervention zones of Anti-malaria Association intervention zones in Amahara National Regional State, namely: Awi, East Gojjam, West Gojjam and South Gondar zones. This study was conducted from January to March, 2011.

Source population: All households in the intervention zones

Study population: people in the selected kebeles

Sampling units: selected Households for the quantitative study

Study units: Individuals in the selected household

\section{Sample size calculation and sampling technique}

Sample size was calculated based on either the prevalence of knowledge, attitude and practice based on the following assumptions:

- $50 \%$ prevalence of knowledge, attitude and practice (p) to get maximum sample size

- $95 \%$ confidence level (CL)

- Maximum tolerable error of $5 \%$ (d)

The sample size was then calculated using the usual single population proportion formula

$$
n=\frac{(Z) 2 P(1-P)}{d 2}
$$

This multiplied by a design effect of 2 , and $10 \%$ non response rate was added and the final calculated sample size was 845 households

Multistage stratified sampling technique was employed to get study subjects. First Anti Malaria Association intervention districts were listed in each zone. Based on size, one district from Awi zone, one district from south Gondar, two districts from west Gojjam and two districts from east Gojjam were selected randomly. Proportional allocation was done based on residence (urban vs. rural) since there is an assumption that there are differences in Knowledge, attitude and Practice among the community towards malaria prevention and control options. From these districts using random sampling technique, three rural kebeles and one urban kebele from five intervention zones and four rural kebele from one district (since there is no urban kebele among the selected intervention kebeles) were selected randomly. From each urban kebele, 28 households and 38 households from each rural kebele were selected using systematic random sampling technique after sampling frame was obtained with the help of kebele leaders, district malaria focal person and local guiders.

\section{Inclusion and exclusion criteria}

People live at least 6 months in the selected kebeles were included in this study and people who live less than 6 months in the Keble and respondents in the households who cannot communicate due to impairment or sever sickness were excluded from this study.

\section{Variables of the study}

Knowledge, attitude and practice of the community on malaria prevention and control options are outcome variables and socio demographic variables, and especial experience to malaria (once contracted malaria, lost family member due to malaria, training on malaria prevention and control, having especial access to information to malaria and other health related issues, leadership role in the kebele).

\section{Operational Definition of Selected Variables}

\section{Knowledgeable}

A study participant whose score is equal to or more than means score of the total questions by the study participants.

\section{Positive attitude}

A study participant is said to have positive attitude towards malaria prevention and control options if his/her score towards the questions is equal to or more than the mean value.

\section{Good practice}

An individual can be considered as having good practice when he/ she practices with a score of equal to or more than the mean value.

\section{Data Collection}

Data was collected using structured questionnaire adapted from standardized questionnaires used by international organizations, national studies such as Demographic and Health survey and published articles in peer reviewed journals. Data was collected by trained data collectors through face to face interview of the respondents. During data collection it has been tried to assess the socio demographic variables, special characteristics of respondents like once contracted malaria, lost family member due to malaria, training on malaria, having special access to information to malaria, participation in Community conversation, leadership role in the kebele, level of knowledge about mosquito behavior, signs and symptoms, treatment modalities and prevention mechanisms, attitude towards malaria prevention and treatment seeking and practices in malaria prevention of the community.

Knowledge assessment part of the questionnaire tries to measure causes of malaria, means of transmission, mosquito breeding site, biting time, signs and symptoms of malaria, signs and symptoms of sever malaria, susceptible groups to malaria, treatment modalities and prevention methods. Attitude assessment part of the questionnaire tries to assess attitude towards malaria prevention and treatment modality options. Similarly, the study tries to assess practices of the community towards malaria prevention.

\section{Data Collection Procedure and Quality Issues}

Data collection was carried out by diploma holder health 
professionals. Appropriate training was given to data collectors and supervisors by principal investigators. To ensure data quality, in addition to training of the research team, checklist was prepared starting from the sample selection to the end of interviewing the respondent. Similarly to get the maximum data quality, local guiders in each kebele and district malaria focal person assisted the data collection process. The collected data was checked on daily bases, and identified problems were corrected as soon as possible by supervisors. A mechanism was developed to bring letter of approval for collected data in the selected areas from the district and kebele administrators and communicate to monitor and witness that the data collectors collect data from the randomly selected households.

\section{Data Management and Analysis}

Data was checked for completeness and consistency, and entered into SPSS version 16 by principal investigators. The data cleaned using frequency and analyzed using SPSS version 16 for windows. The result was presented using simple frequencies with percentages in appropriate tables to display the descriptive part of the result.

\section{Ethical Consideration}

Ethical clearance was sought from Amahara National Regional Health Bureau and permission letter was collected from the respective levels of administrative offices from all levels. Confidentiality and privacy of study participants were maintained. Participants were informed about their right not to participate, not to tell a certain information if they do not want to or even to withdraw without being

\begin{tabular}{|c|c|c|c|}
\hline Variable & Variable category & Number & Percent \\
\hline \multirow{2}{*}{ Residence } & Urban & 133 & 15.6 \\
\hline & Rural & 722 & 84.2 \\
\hline \multirow{2}{*}{ Sex } & Male & 403 & 46.6 \\
\hline & Female & 461 & 53.6 \\
\hline \multirow{2}{*}{ Religion } & Orthodox Christian & 855 & 99.1 \\
\hline & Muslim & 8 & 0.9 \\
\hline \multirow{3}{*}{ Ethnic group } & Amahar & 730 & 84.5 \\
\hline & Awi & 131 & 15.2 \\
\hline & Tgrie & 3 & 0.3 \\
\hline \multirow{4}{*}{ Marital status } & Married & 677 & 78.4 \\
\hline & Single & 63 & 7.3 \\
\hline & Divorced & 69 & 8 \\
\hline & Widowed & 55 & 6.4 \\
\hline \multirow{6}{*}{ Educational status } & Illiterate & 587 & 67.9 \\
\hline & Only read and write & 98 & 11.3 \\
\hline & Primary(1-4) & 62 & 7.2 \\
\hline & Junior (5-8) & 63 & 7.3 \\
\hline & Higher secondary (9-12) & 45 & 5.2 \\
\hline & Above $12^{\text {th }}$ & 8 & 0.9 \\
\hline \multirow{7}{*}{ Occupation } & Farmer & 571 & 66.1 \\
\hline & Housewife & 157 & 18.2 \\
\hline & NGO employed & 2 & 0.2 \\
\hline & Governmental employed & 8 & 0.9 \\
\hline & Daily laborer & 13 & 1.4 \\
\hline & Merchant & 61 & 7.2 \\
\hline & Other* & 52 & 6.0 \\
\hline
\end{tabular}

*Carpenters, students, drivers, who have no work etc.

Table 1: Socio-demographic characteristics of study participants in Antimalaria Association intervention zones, Amahara national regional state, Ethiopia, March, 2011. denied from any possible benefit. Data was collected after obtaining verbal consent from each study participant.

\section{Result of the Study}

The study enrolled a total of 864 household representatives in six districts of Anti-Malaria Association intervention zones of the Amahara National Regional State. These 864 households comprised of 4626 people equaling an average family size of 5.46 individuals. Of these, $626(13.5 \%)$ were under five children, and 95 (2.05\%) were pregnant mothers.

\section{Socio-demographic characteristics of study participants}

Mean age of study participants was 37.84 years. Seven Hundred twenty two (84.2\%) of the respondents were from rural kebeles, about $99 \%$ were orthodox Christians by religion, about $84 \%$ were Amahara by Ethnicity, nearly $85 \%$ were married, and $68 \%$ of the study participants were illiterate. However, $90 \%$ of the households had at least one individual, mostly children, who could at least read and write. Some $66 \%$ of the study participants were farmers by profession. Nearly $54 \%$ of the study participants were females. The table below displays sociodemographic characteristics of study participants (Table 1).

\section{Sources of information about Malaria}

The study indicated that $540(62.5 \%)$ of the respondents got information about malaria from different sources. Health organizations 430 (49.8\%), religious institutions 270 (31.25\%), radio 49 (5.7\%), Friends 44 (5.1\%), Schools 39 (4.5\%) and Television 12 (1.4\%) were major sources of information about malaria in the community. In health institutions, health extension workers were the dominant sources of information about malaria in the community.

\section{Knowledge on mosquito behavior, causes and means of transmission of Malaria}

Six hundred twenty seven $(72.6 \%)$ of the study participants mentioned stagnant water as breeding sites for mosquitoes and only two hundred seventy nine $(32.3 \%)$ of study participants knew that Anopheles mosquitoes are responsible for the transmission of malaria. Four hundred fifty (48\%) had reported mosquito bites human beings at night time and only $14(1.6 \%)$ of the respondents knew that malaria is caused by microscopic organisms (plasmodium species).

The study revealed that misconceptions about causes and transmissions of malaria are very common (Table 2). The three most common misconceptions of study participants about causes of malaria were exposure to cold weather 219 (25.3\%), hunger 135 (15.6\%),

\begin{tabular}{|l|c|c|}
\hline Variables & No (\%) \\
\hline \multirow{3}{*}{ Mosquito breeding site } & Stagnant water & $627(72.6)$ \\
\cline { 2 - 3 } & Running water & $57(6.6)$ \\
\cline { 2 - 3 } & Soil & $21(2.4)$ \\
\hline \multirow{3}{*}{ Mosquito biting time } & Day & $94(10.9)$ \\
\cline { 2 - 3 } & Evening & $242(28)$ \\
\hline \multirow{3}{*}{ Means of transmission } & Night & $415(48)$ \\
\hline \multirow{2}{*}{ Causes of malaria } & Do not know & $30(3.5)$ \\
\cline { 2 - 3 } & Microscopic organism & $212(24.5)$ \\
\hline & Mosquito bite & $279(32.3)$ \\
\hline
\end{tabular}

Table 2: Knowledge of the community about mosquito behavior, breeding site causes and transmission of malaria among Antimalaria Association intervention zones, Amahara national regional state, Ethiopia march 2011. 
Citation: Aderaw Z, Gedefaw M (2013) Knowledge, Attitude and Practice of the Community towards Malaria Prevention and Control Options in AntiMalaria Association Intervention Zones of Amahara National Regional State, Ethiopia. J Trop Dis 1: 118. doi:10.4172/2329-891X.1000118

Page 4 of 7

drinking dirty water 127 (14.7\%). Also the mentioned eating corn cane $27(3.1 \%)$, sleeping with malaria patients $20(2.3 \%)$ and due to evil spirit $10(1.2 \%)$ as causes of malaria.

\section{Recognition of signs and symptoms of Malaria by the community}

The study indicated that three commonly mentioned manifestations of malaria were feeling cold and rigor 557 (64.5\%), fever 325 (37.5\%) and headache 317 (36.7\%). See table below on signs and symptoms of severe malaria by residence.

The study indicated that the loss of consciousness, seizure and vomiting were mentioned by $28.1 \%, 24.4 \%$, and $16 \%$ of study participants as major manifestations of severe malaria (Table 3). Respondents Knowledge on Signs and symptoms of sever malaria by resident in Antimalaria Associations intervention zones, Amahara national regional state, Ethiopia, March 2011 (Table 4).

\section{Perception of the community towards most at risk population groups to Malaria}

Three Hundred twenty four (37.5\%) and 213 (24.7\%) of study

\begin{tabular}{|l|c|c|c|}
\hline \multirow{2}{*}{ Signs and symptoms of malaria case } & \multicolumn{2}{|c|}{ Residence } & Total \\
\cline { 2 - 4 } & Rural & Urban & NO (\%) \\
\hline Feeling cold and rigor & $461(63.85 \%)$ & $96(72.2 \%)$ & $557(64.5)$ \\
\hline Fever & $271(37.5 \%)$ & $54(40.6 \%)$ & $325(37.6)$ \\
\hline Headache & $266(36.8 \%)$ & $50(37.6 \%)$ & $317(36.7)$ \\
\hline Joint pain & $152(21.1 \%)$ & $32(24.1 \%)$ & $184(20.6)$ \\
\hline Vomiting & $130(18 \%)$ & $14(10.5 \%)$ & $144(16.7)$ \\
\hline Sweating & $115(15.9 \%)$ & $24(18.0 \%)$ & $139(16.1)$ \\
\hline Loss of appetite & $86(11.9 \%)$ & $25(18.8 \%)$ & $111(13)$ \\
\hline Thirsty & $85(11.8 \%)$ & $20(15.0 \%)$ & $105(12.2)$ \\
\hline Nausea & $66(9.14 \%)$ & $14(12.6 \%)$ & $82(9.5)$ \\
\hline Bitterness & $67(9.3 \%)$ & $4(2.4 \%)$ & $71(8.2)$ \\
\hline Tiredness & $42(5.8 \%)$ & $10(7.5 \%)$ & $52(6)$ \\
\hline Diarrhea & $27(3.7 \%)$ & $4(2.4 \%)$ & $31(3.6)$ \\
\hline Other* & $90(12.5 \%)$ & $19(14.3 \%)$ & $109(12.5)$ \\
\hline
\end{tabular}

*weight loss, back pain, stomachache, bloody urine, shaking etc

NB: In the table above multiple responses are possible.

Table 3: Respondents Knowledge on signs and symptoms of malaria by district in Anti-malaria Associations' intervention areas, Amahara National Regional State, Ethiopia, March 2011.

\begin{tabular}{|l|c|c|c|}
\hline \multirow{2}{*}{ Signs and symptoms of sever malaria } & \multicolumn{2}{|c|}{ Residence } & \multirow{2}{*}{ Total No (\%) } \\
\cline { 2 - 4 } & Rural & Urban & \\
\hline Loss of consciousness & $194(27.2)$ & $30(22.6)$ & $244(28.3)$ \\
\hline Seizure/Convulsion & $174(24.8)$ & $47(35.3)$ & $211(24.4)$ \\
\hline Vomiting & $118(16.3)$ & $17(12.8)$ & $138(16)$ \\
\hline Unable to eat/drink & $93(12.6)$ & $15(11.3)$ & $108(12.5)$ \\
\hline High grade fever & $80(11.1)$ & $12(9)$ & $92(10.6)$ \\
\hline Dark red urine & $36(5)$ & $9(6.8)$ & $46(5.3)$ \\
\hline Restlessness & $22(3)$ & $5(3.8)$ & $27(3.1)$ \\
\hline Severe Anemia & $23(3.2)$ & $4(2.4)$ & $27(3.1)$ \\
\hline Do not know & $218(30.2)$ & $25(18.9)$ & $243(28.1)$ \\
\hline Others* & $94(13)$ & $19(14.3)$ & $113(13.1)$ \\
\hline
\end{tabular}

* Neck stiffness, Crying all the time, weight loss, back pain, stomachache, bloody urine, shaking etc

NB: In the above table multiple responses are possible

Table 4: Respondents Knowledge on Signs and symptoms of sever malaria by resident in Antimalaria Associations intervention zones, Amahara national regional state, Ethiopia, March 2011.

\begin{tabular}{|l|c|c|c|}
\hline \multirow{2}{*}{ Susceptible group } & \multicolumn{2}{|c|}{ Residence } & \multirow{2}{*}{ Total } \\
\cline { 2 - 3 } & Rural & Urban & No (\%) \\
\hline Under fives & $245(33.9)$ & $79(59.4)$ & $324(37.5)$ \\
\hline Pregnant women & $147(20.4)$ & $13(9.8)$ & $213(24.7)$ \\
\hline Equal for all & $155(21.5)$ & $23(17.3)$ & $171(19.8)$ \\
\hline Elderly & $51(7.1)$ & $23(17.3)$ & $120(13.9)$ \\
\hline Adults & $51(7.1)$ & $9(6.8)$ & $60(6.9)$ \\
\hline HIV career & $4(0.6)$ & $7(5.3)$ & $11(1.3)$ \\
\hline Never sick before & $3(0.4)$ & $3(2.3)$ & $6(0.7)$ \\
\hline Don't know & $166(23)$ & $15(11.3)$ & $183(21.2)$ \\
\hline Other * & $36(5)$ & $8(6)$ & $44(5.2)$ \\
\hline
\end{tabular}

*malnourished, farmers, overloaded workers, people in cold areas NB: In the above table multiple response questions are possible

Table 5: Respondents Knowledge on susceptibility for malaria by residence among Antimalaria Associations intervention zones, Amahara national regional state, Ethiopia, March 2011.

participants were correctly identified under five children and pregnant women as the most susceptible groups of the population in communities to malaria (Table 5).

\section{Treatment seeking behavior of the community to Malaria}

From the total respondents, $780(90.8 \%)$ knew that malaria can be treated. Despite this, about 485 (61.1\%) did not know any kind of antimalaria drug and only 171 (19.8\%) and 109 (13.9\%) mentioned coartem and Chloroquine as Anti malaria drugs, respectively.

A total of 626 under five children were present in 864 households studied. Of these, $176(28.11 \%)$ were reported to have an acute febrile illness within the last 6 months before data collection. Of these 50 (28.4\%) of them were taken to health institutions to seek advice and treatment within $24 \mathrm{hrs}$ of starting the fever and the rest did not taken to health institutions within 24 hours of starting fever. For this, the following factors were mentioned as possible reasons: the illness was mild (28.3\%), financial constraint (21.7\%), work overload of care takers (19.6\%), and distance of health care institutions (12.8\%).

The study revealed that children were taken to health institutions with an average of 2.72 days after the occurrence of febrile illness. According to the respondents, they got drugs for their children $45.1 \%$, from governmental health centers, $27.8 \%$ from health posts and $20.1 \%$ from private hospitals, respectively. However, they also admitted the wide spread utilization of traditional or local remedies of malaria like herbal medicine with butter or honey (35\%), goat blood (15\%), animal fat especially tails of sheep locally known us "lat" with alcohol (12.8\%).

\section{ITN possession and utilization by the community}

From the total respondents, $61.29 \%$ reported that malaria is a preventable disease using bed nets. Further inquiries revealed that of the total 864 study participants, $770(89.1 \%)$ of them claimed that they are currently owned bed net (any kind) in the households. Of these, $652 / 770(84.67 \%)$ of the households possessed functional bed nets

About twenty six percent of the general population uses ITN in the previous date of the survey. Of the total 626 under five children in the study households, 447 (71.4\%) of them were reported that they have been slept under bed net a day before the interview took place. Moreover, of the total 95 pregnant women, 56 (56.95\%) of them were reported to have been slept under bed net a day before data collection.

The study also revealed that traditional methods which are not yet proven scientifically were applied to prevent malaria in the community. 
Nearly $40 \%$ of study participants admitted that they use traditional healers to prevent malaria. These include: eating garlic with green paper (22\%), drink juices of haregerasa (8\%), polishing the floor with ten jut (5\%), drinking endode juice (3\%), never eat vegetables (2\%).

\section{Insecticide residual spraying (IRS) and utilization in the community}

Researchers further assessed the level of knowledge and utilization of indoor residual spray. From the total respondents, only $5.37 \%$ believe that malaria can be prevented using IRS. Study participants were also asked whether indoor chemical spray was performed for their house, and whether their house was plastered after indoor chemical spray. Of the total study participants, $369(42.7 \%)$ reported that indoor chemical spray was carried out within 24 months (mean 2.68 months) before data collection, while $104(12.03 \%)$ of study participants reported that their house was plastered or painted after indoor chemical spray within the last 24 months.

\section{KAP of the community towards Malaria}

The levels of knowledge and attitude of study participants regarding to causes, transmission, and prevention of malaria indicated that $66.3 \%, 50.8 \%$ and $64.8 \%$ of the study participants had been regarded as knowledgeable about causes and transmission, clinical manifestations, and prevention of malaria, respectively. While $78.1 \%, 69 \%$, and $47.1 \%$ of the study participants were considered as having positive attitude towards malaria prevention, treatment, and good malaria prevention practices, respectively.

\section{Discussion}

Community Knowledge on malaria prevention and control options is important and the effort is related to either to environmental management, personal protection or vector control. This study indicated only $32.3 \%$ knew that mosquito bite are responsible for malaria transmission which is consistent with KAP study done in Tripal community of Baigachaek area which revealed that $37.6 \%$ of them knew that mosquito bite transmits malaria [4]. However, this study subject's knowledge about the means of transmission of malaria is lower than studies done in Ethiopia. For instance, surveys done in Ethiopia Gondar (74\%), butajira (48\%), Assosa (48\%) and three towns of Western Ethiopia (43.7\%) revealed mosquito bite for transmission of malaria [5-8]. The findings difference with the current finding may be due to some were done on people in towns which may have good access to information and some were done in highly malarias areas and period that there would be more practices about malaria prevention and control activities. This condition may increase the communities' knowledge about malaria prevention and control activities. On the other side, this study result is higher than the result of Ethiopia national malaria indicator survey of Amahara national regional state which indicated that $26.7 \%$ of the respondents have knowledge on mosquito bite as causes of malaria that may be related with study period difference [9]. Even though there are differences between this study and other study findings, all indicated much effort remain to achieve the malaria control and prevention goals.

This study indicated that only $14(1.6 \%)$ were knew that malaria is caused by microscopic organisms. Similarly, a qualitative study done in Amahara and Oromia national regional states supported this study result [10]. Also a study done in North West Tanzania indicated that only $6 \%$ of the respondents have mentioned plasmodium organisms as a cause of malaria [4]. This low knowledge level of the community may be related with malaria prevention activity gives emphasizes on mosquito related problems rather than the parasite. However, this study result is lower than a studie in Assosa Zone, Ethiopia where 30\% of the respondents were aware that mosquitoes carry disease causing microorganisms [6] and malaria indicator survey 2007 result indicated $41 \%$ respondents reported mosquito bite as a cause for transmission of the disease [9].

Misconceptions are common among the respondents on cause of malaria and understanding these is very important for malaria prevention and control. Exposure to cold weather (25\%), hunger (15.5\%) and drinking dirty water (14.7\%) are commonly mentioned misconceptions about the causes of malaria in the community. This finding is consistence with other similar studies done in different parts of Ethiopia [9,11].

Regarding to the biting time of mosquito, majority (48\%) of the respondents mentioned night as a biting time. This study result is lower than studies done in Assosa and Butajira which indicated that $95 \%$ and $73.2 \%$ of the respondents knew that mosquito bites human beings during night time $[7,8]$. This variation may be due to differences in study settings.

The result of this study indicated that study participants mentioned stagnant water as breeding sites. The study indicated that $627(72.6 \%)$ of the respondents mentioned stagnant water as a breeding site for malaria. This finding agrees with the findings of a study done by Wagari and a qualitative study done in Amahara and Oromia regional states indicated that mosquitoes are mainly believed to breed in stagnant water (71\%) [10].

This study indicated that $37.6 \%$ of the respondents mentioned fever as signs and symptoms of uncomplicated malaria. This study figure is lower than Amahara regional state data as reported by Ethiopian national malaria indicator survey of 2007 which indicated that $50.2 \%$ recognizing fever as signs of malaria [9]. This difference may be related to differences in study period in which ours is done during none malarias season and the later one is in malarias season. This finding was supported by a study done in butajira district southern Ethiopia reported fever, headache and shivering and chills as signs and symptoms of uncomplicated malaria [8]. Also a study done in Swaziland, mid 2007 indicated that symptoms such as headache, high temperature/fever and chills were the three most frequently mentioned signs and symptoms of malaria [12].

Also the present study revealed that unconsciousness (28.3\%), seizure/convulsion $(24.4 \%)$ and vomiting $(16 \%)$ were most frequently mentioned signs and symptoms of server malaria. This finding was supported by a study done in Myanmar which reported unconsciousness and convulsion are most frequently mentioned signs and symptoms of malaria [13].

Regarding to malaria prevention options, $86.2 \%$ of the respondents reported that malaria is a preventable disease which is consistent with a study done by Wagari in Ethiopia indicated $85.7 \%$ and a study in Swaziland indicated $78 \%$ of the respondents Knew that malaria is preventable disease [8]. But this study result is somewhat higher than a study done in Nepal indicated only $50.3 \%$ known that malaria is a preventable disease [12].

Free distribution of ITN in Ethiopia particularly since 2005 has significantly increased the coverage of the intervention [9]. According to this study, eight hundred thirty one $(96.2 \%)$ of the respondents reported that they had heard about mosquito net and $52.8 \%$ of them believes ITN can prevent malaria. This study figure is higher than 2007 
malaria indicator survey which indicated only $38.5 \%$ have heard about ITN [9].

This study indicated that $26.4 \%$ of the general population and $71.4 \%$ of less than five children were slept under ITN in the previous night of the survey. This study figure is higher than Ethiopian studies in Ethiopia like Malaria indicator Survey 2007 of Amahara national regional state (51.8\%) and Eastern Ethiopia (25\%) [9,14]. This is due to the fact that there is time difference between the two surveys and ours. However, this figure is almost equivalent to a study done in Metema area in Gondar (72.9\%) [14]. Despite the study year differences in this study and Malaria Indicator Survey of 2007 Amahara national regional state data there is no great difference on pregnant women ITN utilization which indicated $56.9 \%$ and $52.7 \%$, respectively [9].

The study revealed that only $5.37 \%$ of the study participants had accepted indoor residual spray as malaria prevention method. This study indicated that the acceptance rate is lower, $42.7 \%$ of the participants house had been sprayed with insecticide residual. This figure was higher than the report of Ethiopian MIS 2007 which indicated that $14.2 \%$ of the country and $18.7 \%$ of Amahara regional state residents' houses were sprayed with chemicals [9].

Seven hundred eight $(90.8 \%)$ knew that malaria is a treatable disease. This result agrees with a study in Nepal indicated that among the total respondents, $86.4 \%$ were knew that malaria is treatable [12]. Despite this, the study finding indicated that about 485 (61.1\%) did not know any kind of antimalaria drug, 171 (19.8\%) and 109 (13.9\%) mentioned coartem and Chloroquine as anti malaria drugs respectively.

This study indicated that there are people who has wrong attitude towards malaria prevention and treatment where $39.7 \%$ of the study participants responded wrongly to the item "There is no any effective means to prevent malaria, but God". Moreover, 36.6\% of the study participants agreed with the statement "The best way to treat malaria is to remain at home, drink holy water and pray'. This study indicated that $37.5 \%$ of the study participants recognized fever as signs of malaria. However, this figure is slightly higher than MIS of 2007 report of Amahara national regional state report indicated $25.2 \%$ had febrile illness [9].

The study indicated that $28.11 \%$ of less than five children had febrile illness in the last six months of the survey. Of these, only $28.4 \%$ had been taken to health institutions for treatment of illness within $24 \mathrm{hrs}$ of starting febrile illness. This study result is higher than the report of MIS 2007 of Amahara national regional state that indicated only $15 \%$ of the respondents had sought a prompt treatment for their febrile child [9].

\section{Conclusion and Recommendation}

\section{Conclusion}

- Health and religious institutions are the main sources of information about malaria control and prevention activities in the study community.

- Even though there are improvements on the knowledge of the community about the causes, means of transmission, and clinical manifestations compared to MIS of 2007 in Amahara regional state, it was lower than other studies conducted previously in the country.

- There are common misconceptions on causes, means of transmission, clinical manifestations and prevention practices in the community.
- In this study the ability of the community to recognize fever as signs and symptoms of malaria is lower than Malaria Indicator Survey 2007 of Amahara regional state.

- The study indicated that underfive children, pregnant women and HIV careers were identified by the community as at most risky population groups which is in parallel with the national priority on malaria prevention and control.

- More than half of the respondents did not know any kind of antimalaria drugs that can be currently used in this study.

- This study indicated that there is an improvement on believes that ITN can prevent malaria as compared to MIS 2007 in Amahara regional state.

- The study indicated that lower number of people was slept under ITN in the previous date of the survey as compared to Malaria Indicator Survey 2007 result in Amahara regional state.

- The acceptance rate of the community to IRS had showed improvements from the previous studies in the country as well as MIS of 2007 in Amahara regional state.

- There is an improvement on recognizing Febrile illness as symptom of malaria from the result Malaria Indicator Survey 2007 result in Amahara regional state.

- There is an improvement on treatment seeking for less than five children within $24 \mathrm{hrs}$ starting febrile illness compared with MIS of 2007 in Amahara regional state

- Knowledge, attitude and practice of the community towards malaria prevention and control options.

\section{Recommendation}

\section{Based above conclusion we recommend the following points}

- To achieve the intended plan of the intervention program after three years, it is recommended to focus on common misconceptions about malaria causes, means of transmission and clinical manifestations through community involvement activities as a main strategy.

- The ability to recognize febrile illness as symptom of malaria and treatment seeking behavior of the community for febrile children within $24 \mathrm{hrs}$ are low. Therefore special attention must be give to mothers/care takers of less than five children. This can be done by integrating with ANC, PNC and immunization programs.

- Even though there are improvements on the importance of ITN on malaria prevention and control, ITN utilization rate is still very low. Among many reasons for this, the community uses ITN for other purposes. Therefore, efforts are needed to increase utilization of ITN in the community through community conversation and education. In addition, AMA must put efforts on the regional government to develop rules and regulations on proper utilization of ITN in the community.

- IRS has a negative effect on bees and this may reduce the acceptance rate of IRS. Therefore inter sect oral collaboration is essential between the agricultural sector and AMA to reduce the effect of chemical on bees and to scale up IRS acceptance rate in the community. 
Citation: Aderaw Z, Gedefaw M (2013) Knowledge, Attitude and Practice of the Community towards Malaria Prevention and Control Options in AntiMalaria Association Intervention Zones of Amahara National Regional State, Ethiopia. J Trop Dis 1: 118. doi:10.4172/2329-891X.1000118

\section{Acknowledgement}

We are great full to Bahir Dar University, and Debre Markose University for giving permission to do this research. We use this opportunity to express its deep gratitude to Antimalaria Association with Global Fund, Ministry Of Health, and Amahar Regional Health Bureau for their generous financial support.

At last but not least we want to appreciate the efforts of researchers, supervisors, data collectors and district health office malaria focal persons for their efforts to complete this research.

\section{References}

1. Roll Back Malaria Partnership Secretariat, World Health Organization, Geneva Switzerland.

2. President's Malaria Initiative (2008) Malaria Operational Plan (MOP) Ethiopia.

3. Mazigo HD, Obasy E, Mauka W, Manyiri P, Zinga M, et al. (2010) Knowledge, Attitudes, and Practices about Malaria and Its Control in Rural Northwest Tanzania. Malar Res Treat 2010: 794261.

4. Soan V, Gyan Chand. Knowledge Attitude and Practice towards Malaria in Tribal Community of Baigachak Area, Dindori District (M.P).

5. Knowledge, attitude and practice related to malaria and ITN in Uganda. Base line survey: December 1999-2000.

6. Legesse Y, Tegegn A, Belachew T, Tushune K (2007) Knowledge, Attitude and Practice about Malaria Transmission and Its Preventive Measures among Households in Urban Areas of Assosa Zone, Western Ethiopia. Ethiop J Health Dev 21.
7. Republic of South Africa, Department of Health (2008) Evaluation of Malaria Health Education interventions using Knowledge, Attitude, Practices in South Africa.

8. El-Gayoum SME, El-Rayah E, Giha HA, El-Feki AEA (2009) Knowledge, practices and perceptions which affect acquiring malaria in man-made malarious area in Khartoum State, Sudan. Sudanese J Pub Health 1: 199-209.

9. Ethiopian national malaria indicator survey 2007 (2008) Federal Democratic Republic of Ethiopia, Ministry of health.

10. Addis Continental Institute of Public Health (2009) Qualitative Study on Malaria Prevention and Control in Oromia and Amhara Regional States in Ethiopia. Report Submitted to Academy for Educational Development (AED) and NetMark.

11. Wakgari Deressa, Ahmed Ali, Fikre Enquoselassie. Knowledge, Attitude and Practice About Malaria, the Mosquito and Antimalarial Drugs in a Rura Community. Ethiop J Health Dev 1999.

12. Parajuli K, Ghimire $P$ (2010) Epidemiology of malaria in two Eastern districts of Nepal. Kathmandu Univ Med J (KUMJ) 8: 45-50.

13. Kyawt-Kyawt-Swe, Pearson A (2004) Knowledge, attitudes and practices with regard to malaria control in an endemic rural area of Myanmar. Southeast Asian J Trop Med Public Health 35: 53-62.

14. Sibhtu B (2008) determinant of ownership and organization of ITN for malaria control Gursum District. J Trop Med 2: 7-12. 